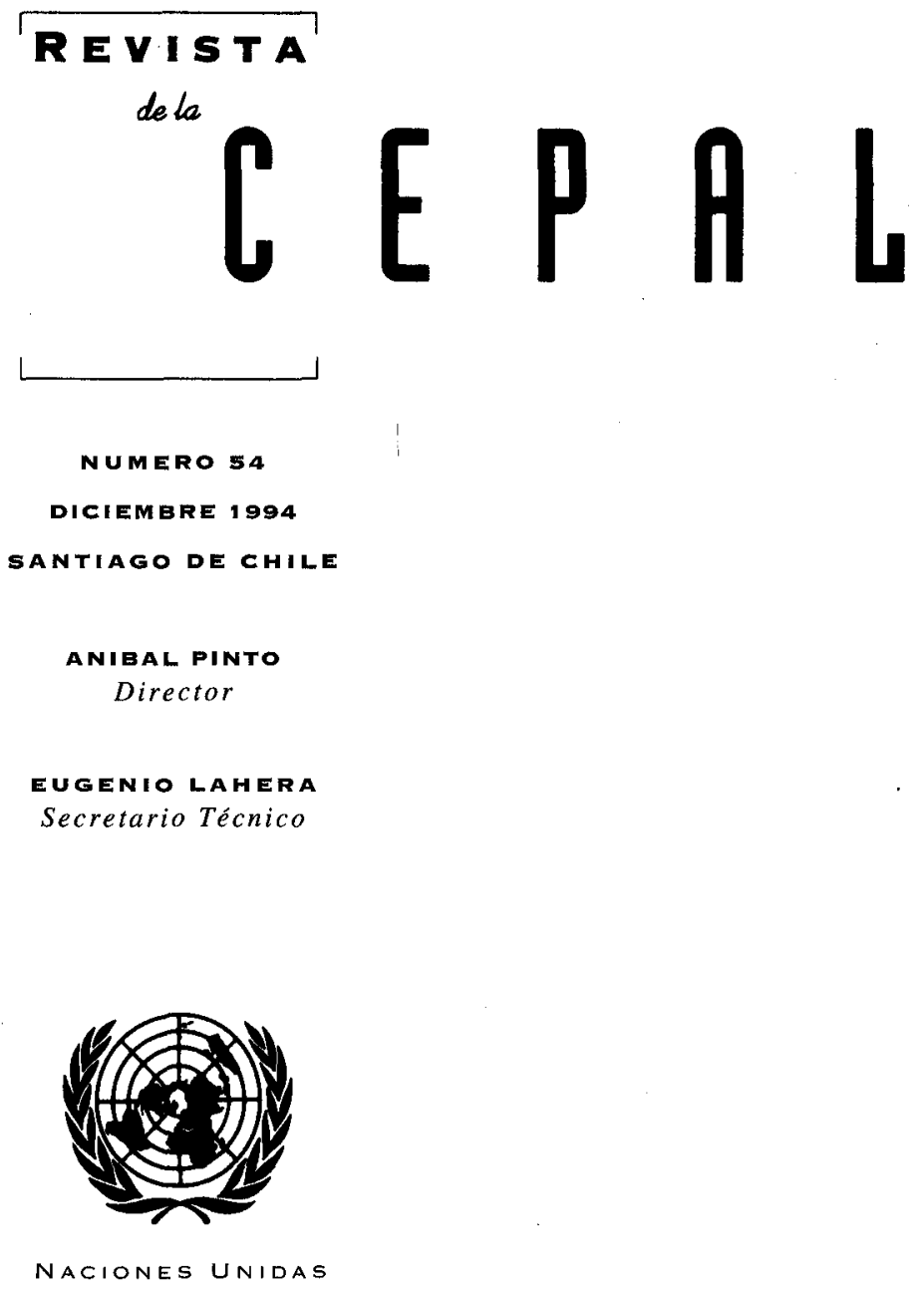


Mercados de salud: morfología, comportamiento y regulación

Jorge Katz y Ernesto Miranda

¿Liberalización o desarrollo financiero?

Günther Held

Ajuste fiscal y gasto social

Rossella Cominetti

Logros y obstáculos en la educación formal de las mujeres

Diane Alméras

¿Qué se entiende por flexibilidad del mercado de trabajo?

Ricardo A. Lagos

Modernización de la supervisión bancaria

Christian Larraín

Costos y beneficios de la integración centroamericana

Luis Cáceres

Algunas enseñanzas de las privatizaciones en Argentina

Daniel Azpiazu y Adolfo Vispo

Ventajas comparativas extraordinarias y crecimiento a largo plazo:

el caso de Ecuador

André A. Hofman y Rudolf Buitelaar

Industria cultural y nuevos códigos de modernidad

Martín Hopenhayn

Orientaciones para los colaboradores de la Revista de la CEPAL

Publicaciones recientes de la CEPAL 


\section{¿Qué se entiende por flexibilidad del mercado de trabajo?}

\section{Ricardo A. Lagos}

Consultor de la Oficina Internacional del Trabajo y Coordinador del Proyecto Regional OIT/FNUAP sobre población, pobreza y mercado laboral en América Latina.
El concepto de "flexibilidad del mercado de trabajo" surgí en el decenio de 1980 en los países industrializados y en algunos países en desarrollo. Tras el deficiente desempeño económico de comienzos de los años ochenta la idea de que la modalidad de funcionamiento de los mercados de trabajo constituía un obstáculo importante al crecimiento económico encontró apoyo entre las autoridades encargadas de formular las políticas, los empleadores y parte de los círculos académicos. Los problemas del mercado de trabajo se achacaron a su "rigidez", que se reflejaba en el precio de la mano de obra, las condiciones de empleo y la cantidad y calidad de los recursos humanos, y que se debería a limitaciones institucionales, políticas sociales, legislación y acuerdos colectivos, negociaciones centralizadas, acción sindical y directrices gubernamentales. Por lo tanto, había que eliminar una serie de normas y reglamentaciones que rigidizan ese mercado, impidiéndole absorber la oferta de mano de obra y adaptarse a las exigencias del cambio tecnológico y la competencia externa. Este artículo examina el significado del concepto de flexibilidad del mercado de trabajo y las diversas formas que puede revestir, y señala que en América Latina los mercados de trabajo se han flexibilizado considerablemente sin que se hayan introducido cambios institucionales importantes. 


\section{I}

\section{Introducción}

El concepto de "flexibilidad del mercado de trabajo" (FMT), así como el debate en torno a él, surgió en el decenio de 1980 en los países industrializados y también en algunos países en desarrollo. Como consecuencia del deficiente desempeño económico de comienzos de los años ochenta — caracterizado por estancamiento y mayores niveles de desempleo e inflación-, la idea de que la modalidad de funcionamiento vigente en los mercados de trabajo constituía un obstáculo importante al crecimiento económico concitó el respaldo de las autoridades encargadas de formular las políticas, de los empleadores en general e incluso de parte de los círculos académicos. La percepción de que habían fracasado los medios tradicionales de luchar contra el desempleo y de lograr la continuación del crecimiento económico (es decir, la ruptura del consenso keynesiano), la comparación del desempeño económico en función de la creación de empleos entre los Estados Unidos y Japón respecto de Europa occidental, la presión de la competencia externa tanto al interior de otros países desarrollados como desde los países de reciente industrialización de Asia, indujo a creer que los problemas radicaban, entre otras cosas, en el funcionamiento del mercado de trabajo. Específicamente, en su "rigidez" en cuanto al precio de la mano de obra, las condiciones de empleo y la cantidad y calidad de los recursos humanos.

Esta rigidez o falta de flexibilidad emanaría de diversos factores: las restricciones institucionales, las políticas sociales, la legislación y los acuerdos colectivos, las negociaciones centralizadas, la intervención sindical y las directrices gubernamentales. Por ende, hay que concentrarse en la eliminación de una serie de normas y reglamentaciones que rigidizan el mer- cado de trabajo, debido a que impiden, por una parte, que este mercado absorba la oferta de mano de obra $y$, por otra, que se adapte a las exigencias impuestas por los cambios tecnológicos y se ajuste a la competencia externa. Desde esta perspectiva, los mercados de trabajo no sólo pasaron a formar parte del diagnóstico de las dificultades económicas, sino también de las medidas correctivas para subsanarlas.

A partir de este diagnóstico básico y sencillo de que se necesitaban relaciones ocupacionales menos rígidas, la conceptualización de lo que se entiende por "flexibilidad del mercado de trabajo" ha conducido a formas diversas y más complejas de flexibilidad. Estas formas representan los diferentes tipos de flexibilidad que necesitan o exigen ciertas economías y los distintos enfoques u opiniones que suscita la flexibilidad.

Por consiguiente, en este artículo se persigue revisar el concepto de "flexibilidad del mercado de trabajo" y determinar las diversas formas que puede revestir, así como la razón de ser de cada una de ellas. Con ese fin, en la sección II se analiza el concepto de flexibilidad. Enseguida en la sección III se examinan las diferentes formas de FMT. En la sección IV se hace referencia a la FMT en América Latina, y se señala que los mercados del trabajo se han flexibilizado sustancialmente, pero sin que se hayan introducido cambios institucionales importantes. Por último, en la sección $\mathrm{V}$ se presentan algunas observaciones finales. Cabe señalar que no se discutirá aquí la conveniencia de una mayor flexibilidad ni si se trata de un aporte que tendrá buena acogida en la economía laboral. Pese al innegable interés de estos últimos temas, ellos rebasan el ámbito del presente artículo que es mucho más restringido (y, ciertamente, menos "exitante").

\section{II \\ El concepto de flexibilidad}

Como sucede a menudo con expresiones utilizadas de

$\square$ El presente estudio se basa en gran parte en un trabajo anterior terminado en marzo de 1992 en la Facultad de Economía y Política de la Universidad de Cambridge, Reino Unido. El autor agradece manera general y amplia, la FMT ha resultado difícil

\footnotetext{
las valiosas observaciones formuladas sobre una versión preliminar por el Dr. Gabriel Palma y el Dr. Paul Ryan. Proceden en este artículo los descargos de responsabilidad habituales.
} 
de definir. Los economistas han aplicado tradicionalmente la palabra flexibilidad a la capacidad que tienen los salarios [nominales] de aumentar o disminuir según la situación del mercado de trabajo (Piore, 1986, p. 146). Sin embargo, a medida que se ha avanzado en el debate sobre la FMT, el concepto de flexibilidad se ha ampliado hasta el punto que actualmente implica la idea de capacidad de adaptación.

De hecho, en la actualidad, este último aspecto es el que ha adquirido mayor importancia cuando se hace referencia al tema de la FMT. Por ejemplo, según la Organización de Cooperación y Desarrollo Económicos (OCDE), la flexibilidad se refiere a la capacidad que tienen los particulares y las instituciones de salirse de las vías establecidas y adaptarse a las nuevas circunstancias (OCDE, 1986a y 1986b, p. 7). Para Michon, la flexibilidad se vuelve imperiosa en un contexto de rápido cambio: denota aptitud para cambiar (Michon, 1987, p. 154). Atkinson sostiene que la flexibilidad alude a cambios en las reglamentaciones y prácticas institucionales, culturales y otras de carácter social o económico que incrementan de manera permanente la capacidad de responder al cambio (Atkinson, 1987, p. 88). Standing afirma que en el plano abstracto, flexibilidad significa capacidad de reaccionar ante la presión y que ser flexible consiste en ser sensible a las presiones e incentivos y poder adaptarse a ellos (Standing, 1986a, p. 6). Para Boyer, la flexibilidad se refiere a la capacidad de un sistema o subsistema de reaccionar ante diversas perturbaciones (Boyer, 1987, p. 113).

Las definiciones anteriores poseen la característica de no comprometerse respecto del contenido real de la FMT. En efecto, no especifican los medios que permitirían adaptarse a las nuevas circunstancias que es precisamente el tema conflictivo en relación con la FMT. Además, esta neutralidad no sólo no prejuzga acerca de los medios sino que deja la puerta abierta a la posibilidad de que la misma necesidad de adaptación pueda satisfacerse mediante formas muy diferentes de flexibilidad (Meulders y Wilkin, 1987, p. 5).

Sin embargo, el análisis de la FMT rara vez se apoya en estos conceptos generales. Desde un punto de vista más específico y restringido, la FMT expresa la noción de que las políticas de empleo deben adaptarse a las variaciones del ciclo económico y al cambio tecnológico. Por ejemplo, la Oficina Internacional del Trabajo (OIT) considera la flexibilidad del mercado del trabajo como la capacidad de éste de adaptarse a las circunstancias económicas, sociales y tecnológicas (OIT, 1986a, pp. 4 a 7). La OCDE entiende la FMT como un ajuste de todos los costos reales de la mano de obra a las variaciones de las condiciones económicas (OCDE, 1986a). Para la Organización Internacional de Empleadores, la FMT es la capacidad de adaptar los costos laborales relativos entre empresas y ocupaciones o la adaptación del costo de los factores de producción a las condiciones del mercado (Organización Internacional de Empleadores, 1985). Por último, para los empresarios estadounidenses, la flexibilidad se asocia con los esfuerzos por transformar la tradicional estructura empresarial sumamente integrada y jerárquica en una forma de organización más dúctil capaz de reaccionar con rapidez ante las cambiantes condiciones del mercado y de la demanda de productos (Piore, 1986, p. 146).

A este nivel más específico y restringido, el concepto de flexibilidad se vuelve a la vez más diverso y sesgado, puesto que las definiciones tienden a concentrarse en los medios específicos para lograr la adaptabilidad de las políticas de empleo a condiciones particulares.

La diversidad se aprecia en la diferencia de acento. Por ejemplo, a pesar de que tanto los Estados Unidos como Europa, tienen en común la importancia que asignan a la búsqueda de relaciones ocupacionales menos rígidas, el concepto de FMT ha adquirido gradualmente significados distintos como consecuencia de los diferentes sistemas de relaciones laborales que predominan en cada país, y por lo tanto, las diferentes necesidades de los empleadores. En efecto, en los Estados Unidos, el debate en torno a la flexibilidad se ha relacionado principalmente con la libertad para distribuir la mano de obra dentro de la empresa. En cambio, en los países europeos, durante el primer lustro del decenio de 1980 se relacionó sobre todo con la libertad de los empleadores para despedir trabajadores en forma transitoria o permanente según las condiciones económicas y, en el segundo lustro, en hacer más compatible la flexibilidad con la seguridad en el empleo. Además, la cuestión de la flexibilidad también ha estado presente en algunos países en desarrollo, donde se ha puesto el énfasis en la reforma de las leyes laborales que afectan la seguridad en el empleo y disminuyen los costos laborales indirectos (Tokman, 1989, p. 39).

Por otro lado, cuando se examinan los distintos criterios sobre la FMT, se observa que el concepto se ha vuelto sesgado. Según uno de estos criterios, la flexibilidad no es mas que la respuesta racional del mercado laboral a las variaciones del entorno económico. La flexibilidad consiste en que la fuerza laboral 
y las horas de trabajo o la estructura salarial se ajusten a las condiciones económicas inestables y fluctuantes. Se sostiene que el ajuste a los cambios es lento e insuficiente debido a las rigideces institucionales, circunstancia que obliga a introducir mayor flexibilidad en las relaciones ocupacionales (OCDE, 1986b).

Según otro criterio, la flexibilidad es un eufemismo para denominar las cambiantes relaciones de poder del proceso productivo y la creciente inseguridad laboral. Es sólo un eslogan de moda que esconde los intentos de revertir los beneficios logrados por los trabajadores durante la posguerra. ${ }^{1}$ La flexibilidad oculta el deseo de los empleadores de hacerse más competitivos mediante el expediente de hacer menos seguras las condiciones de empleo y no a través de la tecnología avanzada o una fuerza de trabajo mejor capacitada. Se sostiene que la flexibilidad tiene el propósito oculto de deprimir los salarios reales, poner término a la protección de los trabajadores y de acelerar el ritmo de trabajo (European Trade Union Institute, 1985).
Sin entrar a analizar lo anterior, baste decir que ambos criterios subrayan aspectos significativos del debate. Por una parte, la necesidad de modificar las instituciones laborales parece justificarse dados los cambios de las condiciones económicas, manifestados en una mayor liberalización del comercio y la globalización de los mercados, el cambio tecnológico, los cambios en la organización de la producción y las nuevas modalidades del flujo internacional de la mano de obra. Por otra parte, la introducción de mayor flexibilidad se ha asociado con efectos negativos para los trabajadores: desigualdad de ingreso, pérdida de puestos de trabajo y diversas maneras en que las condiciones de los trabajadores se volvieron más precarias (Harrison y Bluestone, 1987, y De Bandt, 1991). Estos dos aspectos exigen la elaboración de mecanismos formales (es decir, nuevas reglamentaciones) que reduzcan el costo de la flexibilidad y fomenten al mismo tiempo cambios en las instituciones laborales.

\section{III}

\section{Diferentes formas de flexibilidad}

\section{del mercado de trabajo}

Cabe recordar que la conceptualización de la FMT asume diversas formas, según los aspectos particulares en que se haga hincapié como medio de lograr mayores grados de flexibilidad. A continuación, se hace un intento de determinar esas formas y de explicar sus fundamentos. Teniendo presente lo anterior, se examinan tres tipos diferentes de flexibilidad: flexibilidad de los costos laborales, flexibilidad numérica y flexibilidad funcional.

\section{Flexibilidad de los costos laborales}

De las muchas formas de rigidez susceptibles de impedir el buen funcionamiento de los mercados de trabajo, la rigidez de los costos laborales es la más de-

\footnotetext{
${ }^{1}$ Se afirma que la expresión flexibilidad laboral es en sí misma un término sesgado. La expresión "flexibilidad" tiene una connotación positiva: la capacidad de adaptarse a las circunstancias. A la inversa, sus antónimos "inflexibilidad" o "rigidez" tienen connotaciones claramente peyorativas. En este contexto, si la mano de obra o los mercados de trabajo se vuelven cada vez más flexibles, ello tendría que ser social y económicamente conveniente (Standing, 1986a, p. 2).
}

batida. Por "flexibilidad de los costos laborales" se entiende el grado de sensibilidad de los salarios nominales y los costos no salariales a las variaciones de las condiciones económicas en general (por ejemplo, inflación, productividad, relación de intercambio, demanda) y el desempeño de las distintas empresas. La flexibilidad de los costos laborales, por consiguiente, envuelve dos elementos: los costos salariales y los costos no salariales. El primero es la remuneración bruta pagada a los asalariados. Los costos no salariales o indirectos son las contribuciones y cargos obligatorios pagados por el empleador (por ejemplo, beneficios complementarios, impuestos sobre nóminas, seguros de indemnización a los trabajadores, etc.).

Según sostienen los partidarios de esta forma de flexibilidad, un elemento importante - aunque no el único- tras los altos niveles de desempleo es la rigidez a la baja de los costos salariales. ${ }^{2}$ De hecho, se-

\footnotetext{
${ }^{2}$ Freeman concluye que, para un comportamiento dado de la producción, hay una compensación recíproca significativa entre los
} 
gún una opinión muy difundida, la ausencia de este tipo de flexibilidad explica en gran medida el distinto comportamiento del empleo en los Estados Unidos y Europa después de las dos crisis del petróleo. Se afirma que la inercia de los costos laborales reales en muchos países europeos, a diferencia de los Estados Unidos, fue la causa de que el ajuste principal a la crisis del petróleo y la desaceleración de la productividad revistieran la forma de ajustes cuantitativos (es decir, disminución del empleo) en vez de ajustes de precios (es decir, ajustes salariales) (OCDE, 1986b, pp. 9 y 10; Sachs, 1983).

Además, sobre los costos no salariales —que han aumentado más rápidamente que los propios salariostambién recae cierta responsabilidad por los elevados niveles de desempleo. Puesto que su contribución a los costos laborales totales es significativa, incentivan el reemplazo de la mano de obra por el capital (Sarfati y Kobrin, 1988, p. 8)..$^{3}$

En este análisis, una de las maneras de reducir el desempleo e introducir la flexibilidad salarial es eliminar o reducir las diversas barreras que impiden el equilibrio de los salarios. Entre los obstáculos que se citan con mayor frecuencia, figuran la indización de los salarios, los salarios mínimos garantizados, las subidas prestaciones de desempleo y los costos no salariales elevados. Se afirma que este tipo de flexibilidad constituye una fuente de creación de puestos de trabajo y reduce el desempleo. Se da como fundamento que la baja del costo salarial debería incrementar las utilidades y estimular las inversiones, con la consiguiente creación de más puestos de trabajo. Además, a medida que disminuye el costo de la mano de obra en relación con el costo de otros factores de producción, el coeficiente de sustitución capital/mano de obra debería modificarse en favor de esta última.

La postura que preconiza la eliminación o rebaja de los salarios mínimos se funda en dos consideraciones. En la medida en que los salarios reflejen la productividad individual con mayor precisión (por la reducción o eliminación de los salarios mínimos reglamentarios), se podrían generar algunos puestos potenciales con bajos salarios, con lo que aumentarán las oportunidades de empleo para aquellos marginados del mercado laboral debido a salarios elevados y rígidos.

salarios reales y el empleo en todos los países de la OCDE, al igual que en las industrias dentro de cada país. (Freeman, 1988, p. 77).

3 Estos autores también observaron que los costos no salariales elevados desincentivan la contratación de ciertas categorías de trabajadores, respecto de las cuales son relativamente altos (particularmente los trabajadores jóvenes).
Asimismo, mediante la eliminación o moderación de las exigencias en materia de salario mínimo reglamentario, podría absorberse parcialmente el desempleo juvenil (Clarke, 1985). Se sostiene que en general los trabajadores jóvenes poseen pocas aptitudes directamente aprovechables por un empleador; no están acostumbrados a la disciplina laboral y muestran escaso compromiso con determinada ocupación o industria, lo que origina altos índices de rotación. En estas circunstancias, los trabajadores jóvenes representan mayores costos en materia de contratación, selección y capacitación para los empleadores y, por esto, sus salarios deben reflejar estas diferencias de costos y valor productivo respecto de los de los trabajadores adultos. De otra manera, los empleadores preferirán la contratación de adultos.

Los argumentos expuestos han sido cuestionados por varias razones. En primer lugar, la aseveración de que mayores grados de flexibilidad salarial se traducirán en menores niveles de desempleo suele ser controvertida. En algunos estudios se indica que una baja brusca y general de los salarios contribuiría poco a reducir el desempleo (Boyer, 1988, pp. 237 a 239).

En segundo lugar, se señala que el marco teórico que sustenta el llamamiento a favor de una mayor flexibilidad salarial (es decir, el análisis neoclásico de la demanda de mano de obra) es debatible (Rosenberg, 1989b, p. 396). Rosenberg se remite a un estudio de Bowles y Boyer, donde se construye un modelo macroeconómico que tiene en cuenta la influencia de los salarios en la demanda agregada y la determinación endógena de la producción por hora de trabajo, y llega a la conclusión de que el nivel de empleo puede reaccionar positiva o negativamente a determinados cambios en la escala de salarios reales. ${ }^{4}$ Además, Rosenberg sostiene que los trabajos empíricos sobre el comportamiento de los salarios reales durante el ciclo económico tampoco respaldan la teoría neoclásica de la demanda de mano de obra. La investigación realizada por Michon sobre los movimientos cíclicos de los salarios reales durante el período 1950 1982 en los Estados Unidos, Canadá, Japón, Francia, la [antigua] República Federal de Alemania y el Reino Unido demuestra que no existe necesariamente un comportamiento cíclico de los salarios que sea común a estos seis países. ${ }^{5}$

En tercer lugar, se ha señalado que en algunos

\footnotetext{
${ }^{4}$ Rosenberg (1989b), p. 396, basado en Bowles y Boyer (1988).

5 Rosenberg (1989b), p. 396, basado en Michon (1987).
} 
casos, a pesar de la introducción de mayor flexibilidad, los salarios no bajarían de acuerdo con la oferta y la demanda y podrían existir niveles de salario diferentes para lo que constituye al parecer la misma categoría ocupacional. Lo que parecería una distorsión del mercado de trabajo podría considerarse perfectamente racional por quienes participan en las relaciones ocupacionales. Tal es el caso de las empresas en que los empleadores incurren en costos laborales para contratar, probar y capacitar trabajadores y que desean conservar los trabajadores capacitados para recuperar la inversión efectuada en ellos y, por consiguiente, están preparados para pagarles mayores salarios que los que podrían obtener en otra parte. Cuanto más especializada sea una empresa, tanto más difícil será aumentar el empleo mediante una reducción salarial (OIT, 1989, pp. 7 y 8). En este caso, los empleadores, movidos por su propio interés, pagarán salarios por encima del nivel de equilibrio del mercado.

Existen otros dos casos en que las distorsiones del mercado de trabajo pueden resultar beneficiosas para los empleadores. Puesto que el pago de mayores salarios relativos incrementaría la selección de solicitantes de empleo, mejoraría la calidad de la fuerza de trabajo. Además, el pago de mayores salarios incentiva una mayor productividad de la fuerza de trabajo existente, con lo que disminuyen los costos laborales unitarios (OIT, 1989, pp. 7 y 8).

En cuarto lugar, se ha sostenido que la eliminación de los mecanismos de salarios mínimos impediría el progreso técnico al reducir la presión para modernizar la maquinaria fabril. Esto puede contribuir a un debilitamiento de la competitividad, al cierre de las empresas $\mathrm{y}$, por lo tanto, a un mayor desempleo (Standing, 1986b, p. 14).

En quinto y último lugar, no es terminante la aseveración de que el seguro de desempleo en algunos países representa un factor que impide que los salarios bajen lo suficiente como para generar empleo adicional. Las investigaciones sobre los efectos de este beneficio sobre el desempleo, revelan que las diferencias entre los países en materia de indemnización por desempleo no pueden explicar el gran aumento en la duración del desempleo en Europa, o la enorme elevación de los niveles de desempleo en el Reino Unido, Francia y la [antigua] República Federal de Alemania, en comparación con los de Suecia y los Estados Unidos. ${ }^{6}$

Aunque el debate acerca de la flexibilidad sala-

6 Burtless (1987), p. 155. Citado por Rosenberg (1989b). rial dista de haberse cerrado, pueden detectarse algunas tendencias que apuntan a encontrar la manera de superar la rigidez salarial. Una de ellas es la evasión de las reglamentaciones en materia de salarios mínimos y demás acuerdos salariales reglamentarios, ya sea mediante una reforma legislativa, la no aplicación de las leyes o mediante la utilización de arreglos laborales que escapan a su jurisdicción (por ejemplo, trabajo a domicilio y subcontratación) (Standing, 1986a, p. 31).

La tendencia al control salarial se observa también en los esfuerzos que hacen varios países por obtener acuerdos que estipulan la moderación y la desindización de los salarios. ${ }^{7}$ El objetivo principal de estos esfuerzos es lograr que los incrementos salariales dependan del aumento de la productividad.

Al parecer, se fortalece la tendencia a contar con estructuras salariales de dos o múltiples niveles. En Australia se introdujo en 1987 un sistema de fijación de salarios en dos niveles, en el que pueden obtenerse incrementos salariales hasta un máximo de $4 \%$ si aumentan la productividad y la eficiencia industrial ( $\mathrm{Ri}$ mmer y Zappala, 1988). Han aparecido diversas formas de estructuras de remuneración en dos niveles en Canadá y Estados Unidos, en que los nuevos empleados se contratan con menores salarios o prestaciones que los percibidos por empleados de planta que hacen el mismo trabajo (Walker, 1987).

Además, se están popularizando más las formas experimentales de "salarios participativos". Éstos incluyen planes de remuneración acorde a los resultados, cambios hacia la utilización de sistemas de bonificación en reemplazo de parte del salario monetario, conversión de parte de la remuneración total en un componente flexible vinculado con el desempeño y las características de las empresas. ${ }^{8}$

En algunos casos, el sistema de remuneraciones se ha vuelto más flexible mediante reducciones o congelamientos salariales (disminuciones del salario nominal), a cambio de una mayor seguridad en el empleo ("negociación de concesiones"). A este respecto, vale la pena consignar que según una encuesta europea realizada por la Comunidad Europea, una importante proporción $(51 \%)$ de trabajadores estaría dis-

\footnotetext{
7 Sarfati y Kobrin (1998) suministran pruebas de tendencias en materia de políticas de desindización en Australia, Bélgica, Dinamarca, Italia y España.

${ }^{8}$ Este enfoque refleja las ideas expuestas por Weitzmann (1984), según las cuales los incrementos salariales se vinculan (además de la productividad individual) también con el rendimiento general de la empresa y su fuerza de trabajo.
} 
puesta a aceptar reducciones de salario temporales si la empresa que los emplea se hallara en dificultades, siempre que cuando ésta se recuperara, los trabajadores recibieran un porcentaje correspondiente de las utilidades (Comisión de las Comunidades Europeas, 1985, pp. 1 a 6).

\section{Flexibilidad numérica}

La flexibilidad numérica comprende dos aspectos: ajuste de la fuerza de trabajo y ajuste de las horas laborales. El primero se conoce como "flexibilidad numérica externa" y representa la capacidad de las empresas de ajustar la cantidad de trabajadores (aumentándola o disminuyéndola) para responder a las variaciones de la demanda o a los cambios tecnológicos. El otro, conocido como "flexibilidad numérica interna", trata de la libertad que tienen las empresas para modificar el número de horas laborales sin variar la cantidad de empleados.

\section{a) Flexibilidad numérica externa}

Los argumentos esgrimidos a favor de una mayor flexibilidad numérica externa (o flexibilidad del empleo) se relacionan íntimamente con los mencionados para la flexibilidad salarial. Se afirma que las reglamentaciones destinadas a proteger el empleo son demasiado restrictivas e impiden, por ende, adaptar los recursos humanos a las condiciones del mercado. En esta visión influyen también notoriamente las observaciones sobre el comportamiento del empleo y el desempleo en los Estados Unidos y los países europeos. Se sostiene que los niveles de desempleo relativamente bajos en los Estados Unidos son consecuencia sobre todo de la libertad que tienen los empleadores para despedir trabajadores en forma transitoria o permanente en respuesta a los cambios económicos.

Se estima que una mayor flexibilidad ocupacional se traducirá en más empleo y menos desempleo. La razón en que se sustenta el llamamiento a una mayor flexibilidad ocupacional subraya que las leyes protectoras del empleo, al hacer difíciles y onerosos los despidos, impiden que las empresas contraten trabajadores adicionales si los necesitan, porque saben que no podrán despedirlos fácilmente si cambian las circunstancias (Organización Internacional de Empleadores, 1985; OCDE, 1986a, y OIT, 1986b). Además, las empresas tropiezan con dificultades para establecer formas más flexibles y diversificadas de relaciones contractuales (las llamadas "formas atípicas de empleo"). ${ }^{9}$ Estas cir- cunstancias alientan la sustitución de la mano de obra por el capital. Por lo tanto, las medidas que aminoran las rigideces en materia de despidos (es decir, que los abaratan y facilitan) disminuirán los costos laborales. Esto tendrá un doble efecto: primero, se alienta la sustitución del capital por mano de obra, con lo que se fomenta el crecimiento del empleo. En segundo lugar, un proceso de despido más expedito y menos oneroso debería incentivar a los empleadores a contratar trabajadores adicionales en períodos de crecimiento (Rosenberg, 1989a, p. 11). Además, si se permite a las empresas depender más de las formas atípicas de empleo, podrán ofrecer más puestos de trabajo.

La manera de mejorar la flexibilidad ocupacional consiste principalmente en relajar las leyes de despido o los acuerdos de relaciones laborales que las reglamentan $\mathrm{y}$, en menor grado, en recurrir más a contratos de plazo fijo, trabajo temporal y trabajo a jornada parcial. De hecho, gran parte del debate gira en torno a las reglamentaciones que norman los despidos: preavisos, montos de la indemnización, supremacía del derecho de antiguiedad, acuerdos previos de diversos órganos, etc.

Se han cuestionado los efectos supuestamente positivos sobre el desempleo de este tipo de flexibilidad. Aunque los estudios no son concluyentes, hay pruebas que indican que los efectos de las medidas de flexibilidad laboral en términos de puestos de trabajo son escasos y el análisis más minucioso de la flexibilidad numérica revela incluso un incremento y no una disminución del desempleo (Meulders y Wilkin, 1987, p. 15) ${ }^{10}$

Además, se ha cuestionado también la opinión ampliamente difundida de que las reglamentaciones europeas en materia de despidos son más estrictas. En primer lugar, la correlación entre tasas de desempleo elevadas y mercados de trabajo rígidos no se cumple en todos los casos. Los países nórdicos y Austria han mantenido bajos niveles de desempleo a pesar de tener mercados de trabajo "rígidos". En segundo lugar, se afirma que se ha subestimado el grado de flexibilidad de que disfrutan los empleadores en Europa, en tanto que se ha exagerado el que tienen los Estados Unidos. ${ }^{11}$ Aunque los empleadores estadounidenses tienen libertad para modificar los niveles de empleo según las necesidades del mercado, están

\footnotetext{
${ }^{9}$ Se insta a recurrir más a los contratos de plazo fijo, el trabajo a jornada parcial y el trabajo temporal.

${ }_{10}$ Otros estudios que ponen en entredicho los efectos positivos de la flexibilidad numérica externa sobre el empleo son los de Boyer (1987) y Michon (1987).

${ }^{11}$ Véanse Piore (1986), p. 155; Flanagan (1987), p. 164 [la referencia se tomó de Rosenberg (1989b), p. 396]; Emerson (1987), p. 777; y Rosenberg (1989a), p. 10, nota 10.
} 
obligados a respetar las normas y los procedimien$\operatorname{tos}^{12}$ que hacen muy onerosos los despidos e impiden por tanto nuevas contrataciones. En el caso de las reglamentaciones europeas que norman la seguridad en el empleo - si bien existe una variación importante entre los países-, éstas son mucho menos rígidas de lo estipulado y su efecto principal consiste en demorar el ajuste del empleo y hacerlo más oneroso.

Aún más, se ha sostenido que una relajación de las normas que regulan los despidos propicia un cambio en las modalidades de empleo, en que en desmedro de los trabajadores a jornada completa y permanentes, aumentan los que laboran a jornada parcial, temporales y de plazo fijo, que carecen de una legislación protectora del empleo. ${ }^{13}$ Esto puede llevar a la creación de una categoría de trabajadores cuyas condiciones laborales son generalmente inferiores a las que disfrutan sus pares a jornada completa. ${ }^{14} \mathrm{~A}$ su vez, se afirma que ello conduciría a la institucionalización de un mercado del trabajo dual, que comprendería, por una parte, empleos mal remunerados e inestables y por otra, ocupaciones estables y bien pagadas (Clarke, 1985; Piore, 1986; Standing, 1986b; Meulders y Wilkin, 1987, y OIT, 1989).

\section{b) Flexibilidad numérica interna}

Una mayor flexibilidad en la organización del horario laboral (es decir, la reducción y reestructuración del mismo) se considera una alternativa posible a los ajustes salariales u ocupacionales cuando varían las condiciones económicas. La variedad de aspectos que comprende esta forma de flexibilidad es amplia: fijación de horas de trabajo normales o máximas por semana (mes o año), diversas formas de trabajo escalonado, organización de las horas extraordinarias y del tiempo libre compensatorio, trabajo fuera de horarios autorizados (durante el fin de semana) e ingreso a la fuerza de trabajo o salida de ésta por jubilación (Meulders y Wilkin, 1987, p. 9; OIT, 1989, p. 18; OCDE, 1989, p. 15; Rosenberg, 1989a, p. 9).

\footnotetext{
12 En materias como antigüedad en el empleo, asignación del trabajo en la empresa y distribución de tareas.

13 De acuerdo con algunos estudios, el incremento de estas modalidades de empleo permite sostener que estas formas de FML causan desempleo en vez de solucionarlo. Esta aseveración se basa en la afirmación de que el desempleo crónicamente alto y en aumento ha coincidido con estas formas crecientes de FMT (formas de trabajo ocasionales y temporales, paso de negociaciones colectivas a arreglos individuales, etc.) (Standing (1986a) y Rodgers y Rodgers (1989).

14 Los trabajadores con horas de trabajo o ingresos que no alcanzan ciertos umbrales pueden ser excluidos de muchas de las disposiciones básicas de la legislación protectora o de las prestaciones. OIT (1989), p. 19.
}

El debate acerca de la flexibilidad numérica interna (o flexibilidad del horario laboral) es menos controvertido que el relativo a la flexibilidad salarial y ocupacional. En principio, esta forma de flexibilidad se considera una fuente de beneficios potenciales mutuos para trabajadores y empleadores, que puede contribuir a conciliar parte de sus intereses contrapuestos (Meulders y Wilkin, 1987, p. 9; OIT, 1989, p. 18).

La reducción del horario de trabajo ha sido la forma de flexibilidad de mayor aceptación. Para los sindicatos, coincide con la idea de solidaridad entre los trabajadores en la lucha contra el desempleo y con cierta equidad en la participación en el empleo (Sarfati y Kobrin, 1988, p. 26). Además, la flexibilidad numérica interna coincide con el resultado del cambio de actitud frente al trabajo y la recreación, en que los trabajadores buscan más espacio para organizar sus vidas privadas. ${ }^{15}$

Los empleadores tienen particular interés en ampliar la jornada normal. Dado el alto costo de los nuevos equipos, buscan la manera de que funcionen el mayor tiempo posible, ${ }^{16}$ e introducir de este modo hasta tres o incluso cuatro turnos de trabajo (OCDE, 1989 , p. 15). Además, se afirma que una mayor flexibilidad numérica interna contribuiría a lograr una utilización más eficiente del horario laboral, al permitir una adaptación más precisa de éste a las fluctuaciones de la demanda. ${ }^{17}$

En cuanto a los efectos en el empleo de esta forma de flexibilidad, se afirma que ello depende de los objetivos políticos y sociales en los que se inscribe (Meulders y Wilkin, 1987, p. 9) y, por lo tanto, de la índole de la flexibilidad del horario laboral en cuestión. Por ejemplo, la reducción de la semana laboral puede crear puestos de trabajo o al menos mantener aquéllos que de otra manera desaparecerían, al distribuir los puestos disponibles entre un mayor número de trabajadores. En cambio, al disminuir la edad de jubilación, se reemplazan trabajadores de mayor edad por otros más jóvenes y puede bajar el desempleo por

\footnotetext{
15 Este apoyo se refleja en parte en los resultados de una encuesta a los empleados realizada por la Comunidad Europea. La mayoría de los encuestados aceptaría un horario de trabajo fijado mensualmente $(39 \%)$ o incluso anualmente $(16 \%)$, y uno de cada tres estaría dispuesto a trabajar por las noches y en sábado a cambio de menos horas anuales de trabajo (Comisión de las Comunidades Europeas, 1985, pp. 1 a 6).

16 De este modo los gastos fijos de capital pueden distribuirse en un mayor número de horas y unidades de producción.

${ }^{17}$ Durante las horas diurnas o en ciertos días de la semana o el mes puede variar la intensidad de la actividad comercial, como en el caso de los tiendas minoristas, o durante temporadas más largas, como suele ocurrir en la industria manufacturera. OIT (1989), p. 18.
} 
contracción de la fuerza laboral y no por la creación de puestos de trabajo (Rosenberg, 1989a, p. 12).

A pesar de la actitud positiva respecto de un horario de trabajo flexible, se han formulado algunas críticas. Se sostiene que se corre el riesgo de marginar varias categorías de trabajadores y que pueden surgir conflictos entre los nuevos estilos de trabajo y el ritmo tradicional de la vida familiar, social y escolar, o entre el horario laboral y el tiempo libre (Gaudier, Greve, Grootings y Hethy, 1986, y OCDE, 1986a). Los sindicatos han objetado que los sistemas de seguridad social y jubilación todavía se basan en el empleo a jornada completa durante horas fijas y son incompatibles con un horario de trabajo flexible (European Trade Union Institute, 1985).

\section{Flexibilidad funcional}

La flexibilidad funcional se refiere a la capacidad de una empresa para utilizar eficazmente su fuerza de trabajo variando la labor que desempeña ante modificaciones en el volumen de trabajo y las posibilidades que ofrecen las nuevas tecnologías. Esta forma de flexibilidad se relaciona con la movilidad de los trabajadores dentro de la empresa; entraña la capacidad empresarial de reorganizar los puestos de trabajo. A su vez, ello requiere tener acceso a una fuerza de trabajo capaz de realizar las diferentes tareas del proceso productivo; requiere que los operarios puedan adaptarse a una serie de tareas de diversos niveles de complejidad: aptitudes múltiples, rotación en el empleo, unidades de trabajo, cambios en la división funcional del trabajo, readiestramiento, perfeccionamiento (Meulders y Wilkin, 1987, p. 8). En síntesis, la flexibilidad funcional apela a los conocimientos técnicos y competencia de los trabajadores y a su capacidad de dominar diversos segmentos del mismo proceso productivo (Boyer, 1987, p. 109).

Desde la perspectiva de los trabajadores, la flexibilidad funcional - puesto que no recurre a reducciones salariales o a restricciones de los derechos laborales-es preferible a la flexibilidad salarial $\mathbf{u}$ ocupacional. Este tipo de flexibilidad les permite utilizar mejor sus conocimientos técnicos y competencias, involucrarse más en el proceso productivo $\mathrm{y}$, a la postre, desempeñar un papel más activo en la administración de las empresas. Se le considera una manera de satisfacer las aspiraciones de los trabajadores y, al mismo tiempo, uno de los instrumentos que permite al mercado de trabajo responder a la reorganización de los sistemas productivos (OCDE, 1990, p. 24).
El estímulo para lograr una mayor flexibilidad funcional ha sido principalmente la introducción de nueva tecnología. Puesto que los nuevos equipos tienden a desdibujar las fronteras ocupacionales, las empresas están obligadas a depender de trabajadores con múltiples destrezas que puedan adaptarse a diferentes funciones dentro del proceso laboral. Sin embargo, en algunos casos, el estímulo proviene de la necesidad de reducir los costos; se pueden rebajar bastante los costos si se reestructura el proceso productivo existente y se introducen los cambios correspondientes en la manera de utilizar la mano de obra (OIT, 1989, p. 19).

Además, la flexibilidad funcional se plantea como una manera de manejar un entorno comercial cada vez más incierto (Rosenberg, 1989a, p. 12). Como en algunos sectores la variación de la demanda ha sido mayor - debido a la aparición de modalidades de consumo más diversificadas y especializadas - y las empresas están obligadas a responder a las señales del mercado en lapsos más breves, los recursos deben poder destinarse a nuevos usos. Esto es factible debido a la existencia de una tecnología a la vez moderna y más flexible y a una fuerza de trabajo polivalente con múltiples aptitudes. Estos elementos mejoran la capacidad de los empleadores para manejar situaciones de estrangulamiento o disminución de la producción mediante el desplazamiento de la mano de obra en vez de recurrir a contrataciones o despidos (Rimmers y Zappala, 1988, p. 568).

Por consiguiente, un elemento capital de la flexibilidad funcional es la presencia de trabajadores con múltiples capacidades que les permiten cambiarse de una actividad o puesto a otro. He ahí por qué los programas de capacitación se incorporan crecientemente en las relaciones formales entre empleadores y sindicatos. Aunque se han hecho progresos en la manera en que tanto los empleadores como los trabajadores perciben la capacitación, subsisten algunos problemas. Por ejemplo, no todos los trabajadores aprovechan por igual los nuevos programas de capacitación. Algunos empleadores quizá se muestren renuentes a invertir recursos en capacitar trabajadores mayores, con el argumento de que el período previsto de recuperación de la inversión es menor o que es más oneroso y, por consiguiente, quizá prefieran contratar trabajadores jóvenes (OIT, 1989, p. 21).

Otro problema puesto de relieve es la falta de políticas relacionadas con las regulaciones que eviten que empresas de la competencia se lleven trabajadores —es decir, para impedir que otras empresas atraigan trabajadores recién capacitados sin cargar con el 
costo de su capacitación-, circunstancia que puede disminuir el interés de los empleadores en proporcionar capacitación (OIT, 1989, p. 21).

La flexibilidad funcional depende en gran medida de la reducción de las barreras de demarcación ocupacional. Sin embargo, suelen existir normas explícitas o implícitas sobre los requisitos de dotación de personal y los tipos de trabajo que pueden realizar las distintas clasificaciones ocupacionales, y que por ende estorban el desarrollo de este tipo de flexibilidad. Esto sucede especialmente en los Estados Unidos y constituye la principal preocupación de los empleadores. En efecto, en ese país, la gerencia posee relativa libertad para variar el nivel ocupacional, no así la asignación de trabajo dentro de la empresa. Los empleadores estadounidenses sostienen que, en relación con esta forma de FMT, los mercados de trabajo europeos son más flexibles y ello explica en gran medida por qué se ha estancado la productividad laboral en los Estados Unidos (Piore, 1986, p. 149).

Sin embargo, los sindicatos se muestran a menudo recelosos de los intentos de la gerencia por reducir las barreras de demarcación. La reorganización de la producción y la introducción de nuevas tecnologías puede contribuir a intensificar el trabajo y aumentar la fiscalización administrativa del proceso productivo. Los sindicatos temen, asimismo, que la distribución flexible de la mano de obra aumente la segmentación interna del mercado de trabajo entre una fuerza de trabajo "central" especializada y otra "periférica" no capacitada.

\section{IV}

\section{El mercado de trabajo latinoamericano y la FMT}

Como se señaló en un comienzo, en el decenio de 1980 la idea de que la introducción de mayor flexibilidad en los mercados de trabajo mediante la desregulación facilitaría la adaptación de aquéllos a las nuevas exigencias impuestas por el ajuste estructural, encontró un apoyo considerable. A este respecto, en los países industrializados se ha llevado a cabo un proceso de desregulación tendiente a eliminar los obstáculos institucionales que impiden al mercado de trabajo adaptarse a las nuevas condiciones de producción y competitividad internacionales. Sin embargo, en las regiones menos desarrolladas el mercado de trabajo no está tan regulado y donde sí lo está, las instituciones a menudo son incapaces de hacer cumplir las leyes. En tal caso, es probable que la flexibilidad requerida con fines de ajuste estructural no acarree forzosamente un proceso de desregulación. Se afirma aquí que gran parte de América Latina no constituye una excepción a este fenómeno. De hecho, se puede sostener que el mercado de trabajo ha adquirido una flexibilidad considerable, sin que se haya visto acompañada, ni mucho menos fomentada, por reformas institucionales importantes. Ha sido un proceso de flexibilización sin desregulación. Así las cosas, la receta para conseguir mayor flexibilidad a través de la desregulación no es del todo clara. A continuación, se abordan el proceso de flexibilización de hecho o subterránea y el rezago institucional. ${ }^{18}$

18 El resto de la presente sección se basa en parte en un trabajo

\section{La flexibilización subterránea}

Un análisis global de los acontecimientos en el mercado de trabajo latinoamericano en los años ochenta indica que las tendencias principales apuntan hacia una mayor flexibilidad. Esto es congruente con las conclusiones a que llegó el PREALC (1990) en el sentido de que entre 1980 y 1989 el mercado de trabajo de América Latina se caracterizó por una menor seguridad en el empleo, el reemplazo del empleo a jornada completa por el de jornada parcial y el recurso cada vez mayor a la subcontratación.

Uno de los indicadores de este proceso es la elevada tasa de crecimiento anual del sector informal (6.7\%), es decir, más del doble de la del sector formal $(3.0 \%)$, que pasó de $16 \%$ de la población económicamente activa del sector no agrícola en 1980 a $22 \%$ en 1989.

Otro indicador del aumento de la flexibilidad es la expansión extraordinaria del empleo en la pequeña empresa (aquella con menos de 10 empleados), concentrada en los servicios y la industria. Dentro del sector formal, la tasa de crecimiento del empleo en la pequeña empresa fue de $7.5 \%$, en comparación con sólo $0.5 \%$ en las empresas medianas y

anterior (mimeografiado) elaborado conjuntamente con Eugenio Tironi y titulado Flexibilisation and regulation of the labour market. El documento fue preparado para el Departamento de Empleo y Desarrollo de la Oficina Internacional del Trabajo (EMPLO/OIT), Ginebra. 
grandes. A lo menos $40 \%$ de todas las colocaciones creadas en el área urbana entre 1980 y 1989 obedecí al dinamismo de la pequeña empresa. Su proporción de la población económicamente activa, que era de $15 \%$ en 1980 , subió a $21 \%$ en $1989 .{ }^{19}$

Un tercer indicador es la tendencia - también observada por el PREALC - de las grandes empresas a subcontratar la producción de ciertos componentes básicos de su propio proceso productivo, así como algunos servicios indispensables (como transporte, limpieza, mantenimiento y seguridad) con pequeñas empresas e incluso con algunas de las unidades más modernas del sector informal (PREALC, 1990).

Un cuarto indicador de la mayor FML es el descenso de la tasa de crecimiento del empleo en el sector público. Este fenómeno es importante, ya que se trata de un sector que se ha caracterizado por su gran rigidez. Si se toma el período 1980-1989 en su conjunto, esa disminución no fue apreciable, puesto que sólo bajó de $15 \%$ a $14 \%$ de la población económicamente activa (PREALC, 1990). Sin embargo, si se considera la tasa anual de crecimiento de dicho empleo en los últimos decenios, es evidente que se ha producido una drástica desaceleración. De hecho, descendió de un promedio de $4.5 \%$ en el período 1950 1980, a $3.7 \%$ entre 1980 y 1989. Esta desaceleración resulta aún más evidente si se considera que entre 1980 y 1983 el empleo fiscal todavía crecía a la elevada tasa de $4.7 \%$ anual, aunque disminuyó a una tasa de $2 \%$ anual en el período 1986-1989.

La tendencia hacia una menor absorción del empleo por parte del Estado ha proseguido a ritmos diferentes en los distintos países. Sin embargo, prácticamente todos los países de la región han adoptado programas para reducir el empleo público. Aún es prematuro aventurar cuál será el resultado de estos programas, pero es probable que permitirán reducir algunas de las rigideces del mercado laboral.

Un quinto indicador que pone de relieve el grado de FMT experimentado en la región es la flexibilidad de los costos de la mano de obra. En el decenio de 1980 se deterioraron tanto los salarios mínimos legales como los salarios medios reales, lo que puso de manifiesto una importante flexibilidad a la baja. A comienzos de los años noventa, el promedio de los salarios mínimos en América Latina descendió en al-

\footnotetext{
${ }^{19}$ La tasa de crecimiento de la pequeña empresa debe considerarse con cautela, por cuanto el total calculado por el PREALC (1990) para la región en conjunto acusa fuertemente la influencia de estimaciones de una gran expansión en Brasil.
}

rededor de $35 \% .{ }^{20}$ En cuanto a los salarios reales, cuando se consideran en conjunto sus promedios en los distintos sectores de actividad regional (agricultura, construcción, industria), se advierte que en 1992 todos ellos eran inferiores a los que existían a comienzos de los años ochenta (PREALC, 1993).

Existen también otros indicadores importantes del proceso de flexibilización que tiene lugar en América Latina. Por ejemplo, en casi todos los países se tiende a privilegiar el pago a destajo en desmedro de los salarios por hora, así como el trabajo a domicilio en vez de concentrar trabajadores en fábricas o empresas. Esto coincide con la creciente integración de la mujer al mercado de trabajo, que fue la característica más importante en la oferta de mano de obra del decenio de 1980 (PREALC, 1993).

La conclusión a que lleva lo anterior es que muchos de los cambios que caracterizan la FMT se produjeron realmente en América Latina y el Caribe en los años ochenta. En vista de que este fenómeno no fue acompañado, ni mucho menos alentado, por reformas institucionales importantes, en la mayoría de los casos (con notables excepciones), se trata de un proceso "subterráneo" estimulado por la dinámica interna del mercado de trabajo para responder a ciertos factores macroeconómicos (liberalización del comercio exterior, reducción de los subsidios estatales, privatización), pero sin el liderazgo activo del Estado. En otras palabras, esta tendencia a la flexibilidad es un fenómeno de hecho que se ha producido a menudo no obstante la existencia de instituciones y no por su intermedio. Esto contribuye a explicar el carácter con frecuencia desorganizado, el campo de acción parcial y desigual y los elevados costos que se asocian con este fenómeno, especialmente en relación con el desempleo.

\section{El rezago institucional}

En el último decenio no se produjo prácticamente cambio alguno en las normas e instituciones que rigen el mercado de trabajo de los países de América Latina. ${ }^{21}$ De manera que aun cuando los mecanismos institucionales vigentes no han estimulado ni encauzado el proceso de flexibilización, tampoco parecen haberlo entorpecido. Esto puede explicarse por el hecho de que

20 Colombia, Costa Rica y Paraguay constituyen la excepción a esta tendencia, donde los salarios mínimos reales en las zonas urbanas fueron mayores en 1992 que en 1980. PREALC (1993).

${ }^{21}$ Hay excepciones. Colombia, Chile, Panamá y Venezuela, por ejemplo, modificaron sus códigos del trabajo. Otros países (como Argentina) estudian actualmente la introducción de reformas. 
estos mecanismos ya eran bastante laxos y, en consecuencia, no existía en la práctica ninguna necesidad de proceder a la desregulación, o porque las normas generales destinadas a proteger a los trabajadores, que entrañan ciertas rigideces, solían ser superadas por la propia dinámica del mercado de trabajo. Esta última situación parece encajar en el contexto de América Latina y explica la flexibilización subterránea.

En América Latina el mercado del trabajo está regulado por el Estado, situación que ha permanecido prácticamente invariable. La protección de los trabajadores se funda en la legislación laboral de aplicación universal, más bien que en convenios colectivos a nivel de empresas o ramas de actividad o en contratos individuales de trabajo (Bronstein, 1990). Por ejemplo, respecto de los despidos (área crucial de la flexibilidad), la ley establece múltiples condiciones o requisitos: en algunos casos, no puede despedirse a un trabajador sin que antes se haya realizado una investigación de los hechos en que se fundamenta el despido; en otros, los despidos deben ser aprobados por la autoridad administrativa o deben notificarse a un comité tripartito, que determina si el despido es justo o injusto. Algunas leyes estipulan que en el caso de trabajadores con más de 10 años de servicio, el despido debe ser aprobado por un tribunal laboral. Cuando los despidos se deben a razones económicas, muchos países exigen que sean aprobados por la autoridad administrativa, por comités de conciliación y arbitraje o por comisiones tripartitas.

En todos los países de la región, el peso de la prueba para demostrar que existen razones válidas para el despido recae sobre el empleador. En algunos países, cuando el empleador no puede aportar esta prueba, el despido se anula automáticamente; en otros, se exige al empleador que pague una indemnización. Existe, asimismo, un tercer grupo de países en los que el empleador puede rescindir unilateralmente un contrato de trabajo sin tener que alegar que hay una causa justa, pero se le exige, sin embargo, el pago de una indemnización que puede variar según la causal de despido.

Las situaciones más excepcionales son las de Chile y Brasil. En Chile, a raíz de ciertas reformas laborales promulgadas a comienzos de los años ochenta, no existe restricción alguna para efectuar despidos salvo el costo que supone para el empleador, el cual se incrementó mediante las reformas laborales promulgadas a finales de 1990 y comienzos de 1991. En Brasil, los trabajadores están protegidos por un "Fondo de garantía por años de servicio" (FGTS), financia- do por el empleador. Este fondo, establecido en 1966, los protege en caso de despido, pero concede flexibilidad al empleador en cuanto a la fuerza de trabajo. Sin embargo, últimamente se han promulgado leyes que, aunque no prohíben el despido, lo hacen considerablemente más oneroso para el empleador.

Los últimos cambios introducidos en algunos países, así como los planes de reforma contemplados en otros, buscan indirectamente la manera de facilitar los despidos. Lo hacen, por ejemplo, ampliando la gama de posibilidades en materia de contratos temporales, con lo cual se otorga mayor flexibilidad para suscribir y rescindir los contratos de empleo. Paralelamente, las empresas recurren cada vez más a agencias de empleo temporal o a empresas que prestan servicios temporales, respecto de las cuales sólo recientemente se han dictado disposiciones jurídicas en algunos países o se han introducido en las reformas contempladas en otros. Ambos mecanismos facilitan la suscripción de contratos temporales o de plazo fijo que no están sometidos a las normas de seguridad en el empleo. Las recientes reformas legislativas tratan asimismo de facilitar los despidos colectivos basados en razones económicas o tecnológicas o de fuerza mayor. Sin embargo, con la salvedad de Chile, no ha habido reformas o cambios directos en la legislación sobre seguridad en el empleo.

De manera que, desde una perspectiva formal, el mercado del trabajo latinoamericano estaría estrictamente reglamentado. Además de establecer requisitos rigurosos para el despido, la ley limita el empleo temporal, restringe los sistemas de pago basados en el rendimiento y desincentiva la subcontratación de mano de obra. Salvo algunos países, la región en su conjunto no ha emprendido una reforma sistemática de las instituciones del mercado del trabajo en la dirección de una desregulación. Sin embargo, el marco institucional preexistente no ha podido impedir el proceso de flexibilización que, como se señaló, se ha desarrollado como un fenómeno subterráneo.

\section{Nueva reglamentación del mercado de trabajo}

Inevitablemente, la flexibilización de hecho o subterránea, sin directrices estatales o encauzamiento institucional y que ha solido soslayar la legislación respondiendo a los estímulos directos de la reestructuración económica, ha adquirido algunas características preocupantes. La más grave es, sin duda, su parcialidad, ya que mientras ciertos segmentos del mercado de trabajo han acusado una hiperflexibilidad súbita, otros han logrado "pro- 
tegerse" bastante bien contra esta tendencia. Tal es el caso, por ejemplo, de las grandes empresas en que, además de las reglamentaciones protectoras emanadas de leyes de aplicación universal (por lo demás poco eficaces), los trabajadores disfrutan asimismo de la seguridad inherente a los convenios colectivos. Por otra parte se han flexibilizado más todos los otros segmentos, donde la ineficiencia de las instituciones ha dejado a los trabajadores "protegidos" a merced de las formas más desenfrenadas de flexibilidad.

El regazo de las instituciones del mercado del trabajo latinoamericano frente a la dinámica resultante del ajuste estructural y las nuevas exigencias en materia de competitividad y organización de la producción, presentan algunas consecuencias graves. Estas deficiencias han alentado una dualización creciente del mercado del trabajo en toda la región; es preciso superar el regazo mencionado para revertir esta tendencia, a fin de encarar los problemas que plantean los segmentos del mercado del trabajo donde las rigideces de tipo corporativo atentan contra una ma- yor productividad, así como reglamentar la flexibilidad que se ha desarrollado en otros segmentos y que ha dejado a los trabajadores desprotegidos. La superación de estas fallas en las instituciones vinculadas con el mercado de trabajo de América Latina no significa necesariamente respaldar las teorías en pro de la desregulación. Tal enfoque sólo tiene sentido cuando las regulaciones existentes constituyen realmente un obstáculo para la flexibilidad, y no está del todo claro que tal sea el caso de América Latina. De modo que la tarea que enfrenta la región es muy distinta: la nueva regulación del mercado del trabajo; en otras palabras, un reacondicionamiento de las instituciones laborales que, además de ampliar la flexibilidad a todos los segmentos de aquel mercado, garantice igualmente a todos ellos un nivel básico de protección. Fundamentalmente, ello supone lograr las condiciones para impulsar la productividad, manteniendo al mismo tiempo ciertos niveles mínimos de protección que establezcan límites sociales aceptables para la reforma económica.

\section{Observaciones finales}

Las diferentes formas de flexibilidad examinadas reflejan los distintos enfoques o criterios sobre el tema de la flexibilidad del mercado de trabajo. En realidad, el debate ha girado en torno a dos perspectivas distintas. Una, denominada defensiva, hace hincapié principalmente en reducir los costos salariales y no salariales (flexibilidad de los costos de mano de obra) y en facilitar el ajuste de la fuerza de trabajo a las fluctuaciones de las condiciones del mercado y los cambios técnicos (flexibilidad numérica externa). Como reacción a este enfoque de la flexibilidad, ganó progresivamente terreno la idea de una flexibilidad ofensiva o proactiva. Este enfoque ofensivo, sin descartar la necesidad de flexibilizar más el mercado de trabajo, pone en tela de juicio muchos de los supuestos basados en la "rigidez" y subraya la necesidad de proporcionar a la fuerza de trabajo capacitación y nuevas destrezas para facilitar su capacidad de adaptación a los cambios del proceso productivo.

El primer enfoque se basa en la opinión de que los mercados del trabajo están excesivamente regulados y, por consiguiente, distorsionados; de ahí la necesidad de desregularlos. En tanto, el enfoque ofensivo reconoce la necesidad de flexibilizar más las relaciones laborales, pero sin reducir la seguridad en el empleo y enfatizando aquellas formas de flexibilidad que los actores sociales consideran menos conflictivas o no conflictivas (flexibilidad numérica interna y flexibilidad funcional).

Sin embargo, interesa señalar que, en la medida en que se ha ampliado el debate, se ha superado sustancialmente la distinción entre flexibilidad defensiva y ofensiva, ya que se ha alcanzado cierto grado de consenso respecto de ciertos temas. En efecto, los enfoques sobre la materia han tendido a pasar de criterios inmediatistas y coyunturales a soluciones de más largo plazo (y menos conflictivas). Existe la percepción de que los problemas estructurales que causan, entre otras cosas, gran desempleo no se superarán recurriendo a medidas que mitiguen temporalmente el ajuste (por ejemplo, flexibilidad salarial, flexibilidad numérica externa). Este punto se ilustra al observar, por ejemplo, la lectura que hace la OCDE - partidaria entusiasta de las formas defensivas de flexibilidad a mediados de los años ochenta- al respecto. Según esta Organización, cuanto mayor sea la flexibilidad del mercado de trabajo, tanto menor será el costo económico del ajuste: habrá menos desem- 
pleo y menos pérdida de producción. La teoría microeconómica estándar postula que, en un mercado de trabajo perfectamente libre, los salarios y el desempleo se ajustan para rectificar los desequilibrios entre la oferta y la demanda. Sin embargo, en el mundo real hay obstáculos que entorpecen el ajuste. Estos exigen la formulación de políticas pertinentes que no sólo ayuden a los más afectados, sino que también fomenten la flexibilidad de las estructuras salariales y el ajuste de los recursos humanos (OCDE, 1984, p. 29).

En otra publicación sostiene que el nuevo marco normativo elaborado por esa Organización exige desviar la atención de los problemas coyunturales y concentrarse en otro basado en la necesidad estructural de que los mercados de trabajo deben ser capaces de reaccionar de manera eficiente ante el cambio económico y social. Deberán fomentarse los mercados de trabajo eficientes, mejorando la calidad de la fuerza de trabajo y reduciendo las desigualdades en el acceso al empleo y la capacitación. Fomentar el funcionamiento eficiente del mercado no significa sencillamente liberar las fuerzas del mercado mediante una desregulación indiscriminada o un criterio estrecho acerca de la flexibilidad salarial (OCDE, 1992, p. 12).

Por último, conviene señalar que el tema de la
FMT también es relevante en los países en desarrollo. En un contexto internacional, la introducción de flexibilidad en el mercado del trabajo tendrá alcance y naturaleza diferentes de un país a otro, según la estructura de la economía, los mecanismos institucionales predominantes en materia de relaciones laborales y el grado de diálogo entre los actores sociales. Dado que el debate acerca de la FMT ha tenido lugar casi exclusivamente en los países industrializados y en relación con ellos, es probable que parte (cuando no la mayoría) de sus análisis y prescripciones no tengan adecuadamente en cuenta o reflejen las diferencias estructurales de los países en desarrollo. En particular, la debilidad de los sindicatos, las bajas tasas de sindicalización, la gran proporción de la fuerza de trabajo que participa en actividades informales y que, por lo tanto, queda fuera del marco institucional, las altas tasas de subempleo y los mercados del trabajo poco reglamentados en relación con los de los países industrializados. Esto es lo que sugiere en parte el examen de la situación en América Latina. En este contexto, entonces, poner el acento en la desregulación de los mercados del trabajo como medio de alcanzar mayor flexibilidad laboral, como sucede en los países industrializados, plantea ciertas dudas.

(Traducido del inglés)

\section{Bibliografia}

Atkinson, J. (1987): Flexibility or fragmentation? The United Kingdom labour market in the eighties, Labour and Society, vol. $12, \mathrm{~N}^{\circ} 1$, enero.

Bowles, S. y R. Boyer (1988): Labor discipline and aggregate demand: A macroeconomic model, American Economic Review, vol. $78, \mathrm{~N}^{\circ} 2$. Princeton, New Jersey, American Economic Association, mayo.

Boyer, R. (1987): Labour flexibilities: Many forms, uncertain effects, Labour and Society, vol.12, $\mathrm{N}^{\circ} 1$, enero. (1988): Defensive or offensive flexibility?, R. Boyer (ed.), The Search for Labour Market Flexibility, Oxford, Reino Unido, Clarendon Press.

Bronstein, A. (1990): Protection against unjustified dismissal in Latin America, International Labour Review, vol.129, $\mathrm{N}^{\circ} 5$.

Burtless, G. (1987): Jobless pay and high European unemployment, R. Z. Lawrence y C.L. Schultze (eds.,), Barriers to European Growth: A Transatlanctic View, Washington, D. C., The Brookings Institution.

Clarke, O. (1985): Labour market flexibility: Two sides of the coin, Social and Labour Bulletin, $\mathrm{N}^{\circ} 3-4$, marzo.

Comisión de las Comunidades Europeas (1985): European Economy, $\mathrm{N}^{\circ} 10$, Supplement B, Luxemburgo, octubre.
De Bandt, J. (1991): Flexibility versus rigidity: The implications, true and false, Labour Market Programme Discussion Papers, $\mathrm{N}^{\circ} 31$ Ginebra, Instituto Internacional de Estudios Laborales (IIEL).

Emmerson, M. (1988): Regulation or deregulation of the labour market, European Economic Review, No 32, Amsterdam, North-Holland, marzo.

European Trade Union Institute (1985): Flexibility and Employment: Myths and Realities, Bruselas.

Flanagan, R.J. (1987): Labour market behaviour and European economic growth, R.Z. Lawrence y CL. Schultze (eds.), $B a$ rriers to European Growth: A transatlantic View, Washington, D.C., The Brookings Institution.

Freeman, R.B. (1988): Labor markets institutions and economic performance, Economic Policy, vol. 6, Gran Bretaña, Centre for Economic Policy Research/Maison des Sciences de l'Homme, abril.

Gaudier, M., R. Greve, P. Grootings y L. Hethy (1986): Management-work-leisure: A review of research trends in Western and Eastern Europe, Bibliography Series, $\mathbf{N}^{\circ} 11$, Ginebra, IIEL.

Harrison, B. y B. Bluestone (1987): The dark side of labour market flexibility: Falling wages and growing income inequality in America, Labour Market Analysis Working Paper, 
$\mathrm{N}^{\circ}$ 17, Ginebra, Oficina Internacional del Trabajo (OIT), Programa Mundial del Empleo, Labour Market Analysis and Employment Planning.

Meulders, D. y L. Wilkin (1987): Labour market flexibility: Critical introduction to the analysis of a concept, Labour and Society, vol. $12, \mathrm{~N}^{\circ} 1$, enero.

Michon (1987): Time and flexibility: Working time in the debate on flexibility, Labour and Society, vol. 12, $\mathrm{N}^{\circ} 1$ enero.

OCDE (Organización de Cooperación y Desarrollo Económicos) (1984): OECD Observer, $\mathrm{N}^{\circ} 127$, París, marzo.,

(1986a): Labour Market Flexibility - A Report by a HighLevel Group of Experts to the Secretary-General, París, mayo. (1986b): Flexibility in the Labour Market - The Current Debate, París, octubre.

(1989): Labour Market Flexiblility - Trends in Enterprises, París, marzo.

(1990): Labour Market Policies for the 1990s, París, enero. (1991/1992): OECD Observer, $\mathrm{N}^{\circ}$ 173, París, diciembreenero.

OIT (1986a): Labour Market Flexibility - Report of an Experts Group Meeting, Ginebra, mayo.

(1986b): Flexiblité du marché de l'emploi, Le monde du travail change: Les problemes de demain, Report of the Director General to the 72nd Session of the International Labour Conference, Ginebra.

(1989): Adjustment, Employment and Labour Market Policies (GB.244/CE/4/3), Commitee on Employment, Ginebra, noviembre.

Organización Internacional de Empleadores (1985): Adapting the Labour Market, Ginebra, septiembre.

Piore, M.J. (1986): Perspectives on labour market flexibility, Industrial Relations Journal, vol. $25, \mathrm{~N}^{\circ} 2$, primavera.

PREALC (Programa Regional del Empleo para América Latina y el Caribe) (1990): Empleo y equidad: Desafío de los 90, Documentos de trabajo, $N^{\circ} 354$, Santiago de Chile, oIT.
(1993): PREALC Newsletter, $\mathrm{N}^{\circ} 32$, Santiago de Chile, septiembre.

Rimmer, M. y J. Zappala (1988): Labour market flexibility and the second tier, Australian Bulletin of Labour, vol. 14, $\mathrm{N}^{\circ} 4$, septiembre.

Rodgers, G. y J. Rodgers (1989): Precarious Jobs in Labour Market Regulation - The Growth of Atypical Employment in Western Europe, International Labour Organization, Ginebra.

Rosenberg, S. (1989a): Labor market restructuring in Europe and the United States. The search for flexibility, S. Rosenberg (ed.), The State and the Labor Market, Nueva York, Plenum Press.

(1989b): From segmentation to flexibility, Labour and Society, vol. 14, $\mathrm{N}^{\circ} 4$, octubre.

Sachs, J. D. (1983): Real wages and unemployment in the OECD countries, Brookings Papers on Economic Activity, $\mathrm{N}^{\circ} 1$, Washington, D.C., The Brookings Institution, mayo.

Sarfati, H. y C. Kobrin (eds.) (1988): Labour Market Flexibility. A Comparative Anthology, Londres, Gower Publishing Company.

Standing, G. (1986a): Labour flexibility: Towards a research agenda, Labour Market Analysis Working Paper, $\mathrm{N}^{\circ} 3$, Ginebra, Labour Market Analysis and Employment Planning, Programa Mundial del Empleo, oIT.

(1986b): Labour flexibility: Cause or cure for unemployment?, Public Lectures Series, N² 25, Ginebra, IIEL.

Tokman, V. E. (1989): Economic development and labor market segmentation in the Latin American periphery, Journal of Interamerican Studies and World Affairs, vol. 31, Nos 1 y 2 , Beverly Hills, California, University of Miami, primaveraverano.

Walker, J. (1987): Two-tier wage systems, Research and Current Issues Series, $\mathrm{N}^{\circ}$ 51, Kingston, Industrial Relations Centre/ Queen's University.

Weitzmann, L. (1984): The Share Economy: Conquering Stagflation, Massachusetts, Harvard University Press. 